\title{
Study on Modeling of Local Intelligent Traffic Scheduling
}

\author{
Chao Zhang ${ }^{1, a}$, Shoujin Wang ${ }^{1, b^{*}}$ and Zhaoyang $\mathrm{Xu}^{1, \mathrm{c}}$
}

School of Information \& Control engineering, Shenyang Jianzhu University, Shenyang, China

a356396059@qq.com, b23917240@qq.com, ${ }^{\mathrm{c} 9} 905777203 @ q q . c o m$

Keywords: Local intelligent transportation; Road modeling; Traffic modeling; Vehicle scheduling

\begin{abstract}
In order to alleviate the congestion of urban traffic roads, reduce the tension of congestion and reduce travel time, through traffic simulation, we can predict and evaluate the actual traffic situation, so as to layout the traffic control facilities preferably. Through the dynamic transformation and optimization of the traffic control parameters and the simulation of road traffic of multiple junctions in all directions or simulation under different conditions, this paper will evaluate the rationality of a few conditions such as road intersection.
\end{abstract}

\section{Introduction}

In recent years, with the expansion of first-tier city scale such as Beijing, Shanghai, Guangzhou and Shenzhen and the substantial increasing of the number of motor vehicles, the average load in the morning and evening peak downtown road network has risen to $95 \%$, and it is hard for relying on the limit line, flow and other coercive to solve a considerable part of the section and crossing the jam in the urban area, the average vehicle speed is generally low, the city traffic problem has become increasingly prominent[1]. At the same time, because of congestion, the number of vehicles start and stop increases, and the energy consumption time is prolonged. Therefore, energy consumption will rise sharply, thus aggravating environmental pollution.

At present, urban traffic facilities in China are developed faster and faster, urban roads are widening, overpasses are increasing, but the problem of urban congestion is still not well solved. In this case, it is necessary to apply the intelligent transportation system to the daily traffic operation. According to the specific situation of the city modernization management to realize traffic operation through more high-tech means, so as to better guide and limit the traffic flow, the effective use of further development for City Road creating environment, driving safety and improve the city road traffic flow rate and reasonable allocation. So as to alleviate the traffic jams, reduce traffic accidents, congestion and reduce travel time.

To solve the problems above, according to the initial input condition traffic, the system will generate traffic simulation prediction and real-time demonstration to the screen, make an assessment on performance of traffic signal scheduling scheme, so as to find out the most suitable for the current road traffic scheduling scheme, to reduce the blind set traffic lights caused by traffic efficiency loss, alleviate traffic congestion[2]. So as to reduce traffic accidents, increase traffic safety, ease traffic congestion, improve traffic efficiency and bus efficiency, reduce traffic load and pollution, and save energy consumption.

\section{Domestic and International Related Research}

International Related Research. In order to solve the problem of vehicle congestion, the United States began to carry out the study of intelligent transportation system in 80s. At present, the application of intelligent transportation system in the United States has covered more than $80 \%$ of the transport facilities, related products are more advanced[3]. The US Department of transportation estimates that the use of intelligent transportation systems have eliminated about 1 million and 200 thousand traffic accidents per year, saving tens of thousands of lives.

Most of the European countries is very small, so the research of intelligent transportation system in Europe to take the entire European integration policy, the organization for economic cooperation and development in order to promote the development of intelligent transportation system and the 
effective coordination of international cooperation across Europe, brings intelligent transportation system into the starting 1986 "Eureka" joint research and development program, aims at establishing a trans European intelligent road network[4].

Japan, with a population of 127 million, is a land scarce country with a large population[5]. There are hundreds of millions of vehicles on the road every day, traffic problems such as traffic congestion, environmental pollution are serious, it is more difficult for Japan to rely on the construction of new road network to solve this kind of problem, so the development of intelligent transportation system, effective use the existing road resource, is the key to solve the problem and the only way which must be passed.

South Korea's Kwangju is a intelligent transportation system demonstration project site, costs 10 billion won. Its construction and application of selected traffic responsive signal system, bus passenger information system, dynamic route guidance system, automation management system, timely broadcast system, electronic toll collection system, parking forecast system, dynamic focusing system, intelligent transportation system center and other 9 items[6].

Singapore intelligent transportation system construction focused on advanced city traffic management system[7]. this system has the traditional functions, Ru signal control, traffic detection, traffic guidance, including permanent electronic traffic control card hit fly. During peak hours and congested roads, tolls can be increased automatically, the use efficiency of the road can be controlled as reasonably as possible.

Australia has earlier engaged in research of intelligent traffic control technology, its construction includes advanced traffic control systems, remote signal system, microcomputer based traffic control system, signal system, road vehicle monitoring system and public information service system. The most famous optimal adaptive traffic control system is used in almost every city in Australia.

Domestic Related Research. The origin of intelligent transportation system in China can be traced back to the end of the 1970s city traffic signal control experimental research, which began to develop rapidly in the middle and late 1990s[8]. In 1995, intelligent transportation system Engineering Research Center of Ministry of communications of the global positioning system "and" Research on GPS road vehicle management system based on "project, in addition the Ministry of communications and various ministries jointly launched the" network environment system "research work. By the end of 2015, ETC has implemented a national network, Chinese ETC completed a total of more than 1.2 lanes, more than 5 artificial swipe lane, about 21 million 715 thousand ETC users, self built more than 1100 service outlets, the rational agent network of about 16 thousand, about 27 thousand of all kinds of service terminal, ETC bus use rate of not less than $25 \%$ of non cash payment the utilization rate of $20 \%$.

In transportation, one of the focuses of the development of intelligent transportation system is to solve environmental pollution and other issues, and meet the requirements of ecological civilization construction[9]. Therefore, in order to meet the increasing traffic demand more than one billion people, double balance the demands of economic development and environmental protection, we need to take a new road traffic development China socialism, thus the use of road traffic with existing infrastructure, with the rapid development of science and technology, based on the establishment of the framework of intelligent transportation system system in China. The key to improve the ability of technological innovation, strengthen the technical standardization work, promote the integration of industrial chain, to achieve the strategic objectives of the modernization of transportation construction.

\section{Main Research Contents}

Intelligent traffic has been developed for many years, but there are still many deficiencies. This paper makes a more in-depth simulation study of the local intelligent transportation, in order to achieve a more reasonable urban traffic scheduling scheme.

Virtual city road modeling. Through two-dimensional modeling of virtual technology, simulate urban roads, buildings and different weather, etc. according to a set of local scenarios. 
Virtual road network. Through reasonable data and information, the road and the road in the virtual environment are connected by inputting signals to form a road network

Virtual vehicle movement. According to the prior statistical traffic condition, entering initial condition, generate the future traffic forecast and demonstrates it on the screen in real time.

The realization of the virtual system. Based on traffic conditions (such as flow rate, density and velocity), changes with time and space travel time, cost, delay, the best route between two points on the distribution of traffic flow re planning, the performance of the traffic signal scheduling scheme to make an assessment, so as to find out the traffic scheduling scheme for the current traffic, reduce the blind set traffic lights caused by traffic efficiency loss, alleviate traffic congestion.

\section{System Modeling}

Build Road Model. Road model is the basis of traffic simulation, forming the basic framework of traffic vehicle operation, and always acting on the simulation process. Therefore, the organizational structure and attributes of the road network have a great impact on the operation of the actual traffic simulation system. It is much significant to build a reasonable and effective road model.

Road Coordinate System. The coordinate system commonly used includes rectangular coordinate system and curvilinear coordinate system in road design, and the two coordinate systems can be converted to each other. A series of characteristics and characteristic points of a road, such as the center and edge lines of a road, that can be defined at the same time by the coordinate points in the two coordinate system. The direct coordinate system is the geodetic coordinate system, and the $S$ in the curvilinear coordinate system is defined as the displacement along the road reference line. $\mathrm{L}$ is defined as the distance to the reference line, and the left direction is along the reference line. $\mathrm{S}$ can be expressed as coordinate points (Xi, Yi), as follows:

Corresponding to each coordinate point ( $\mathrm{Xi}, \mathrm{Yi})$, there is a unique $\mathrm{L}$ corresponding to it, so it is easy to establish a one-to-one correspondence between the Cartesian coordinate system and the coordinate point of the curvilinear coordinate system.

Intersection Structure Definition. In the real world, intersections are nodes that connect two or more sections of the road, which are key components of the road network. The intersection of vehicles in the intersection has complicated spatial relations, and different lanes of different roads are connected through a number of channels. In the virtual environment, the intersection defines the connection between the lanes of each road, and guides the vehicle through the intersection through the virtual lane inside the intersection, see Fig.1.

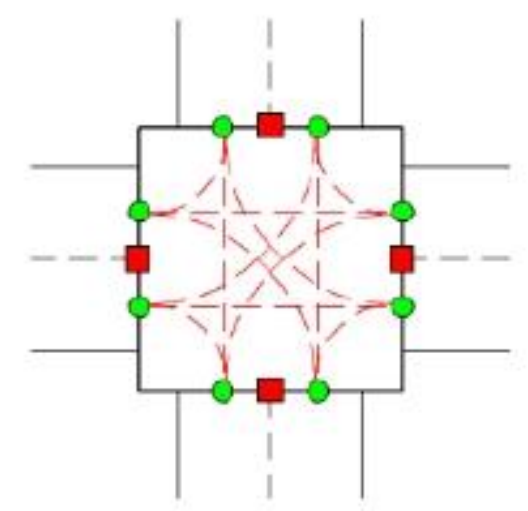

Figure 1 Intersection structure of the road

The red box is the connection point between the intersection and each road. The green dot is the connection point between each lane center line and the intersection. The red dotted line is the inner channel of the intersection, and the red dotted line is connected with the corresponding green dot.

Traffic Lights Setting. Because the traffic flow in each intersection of rush hours, holidays and usual are different, so multi-period and multi-program algorithm are used for the traffic lights 
setting. Traffic signal control system can be divided into single intersection control, trunk control and network control.

The length of a cycle is the time required for a signal to run a cycle, which is equal to the sum of the time of the green light, yellow light, and red light. General lights have the shortest cycle, otherwise can not guarantee a few directions of the vehicle smoothly through the intersection. The longest period is not more than 120 seconds, otherwise will cause the complaint of the waiting driver or the mistake of the signal lamp. The appropriate cycle length is important to the traffic volume at the intersection and to the waiting time of the vehicle.

In traffic control, in order to avoid the intersection of each direction of traffic flow conflict between the method of traffic is usually used in a certain period of time for a cycle, the intersection of a few or the traffic flow is right, and the conflict with other traffic impassable.

The phase can be represented by a directed line segment, and the arrow direction of the directed line is in accordance with the direction of the vehicle motion. Fig. 4-1 is a diagram of a 2 phase system of an intersection, in which first phase north-south traffic flows straight, and the second phase flows straight to East and west. Figure 4-2 is a 4 phase system, first phase north-south traffic flow straight and allow the right, second phase north-south traffic flow left, third phase traffic flow straight and allow things right, fourth things to the left turn traffic flow phase.
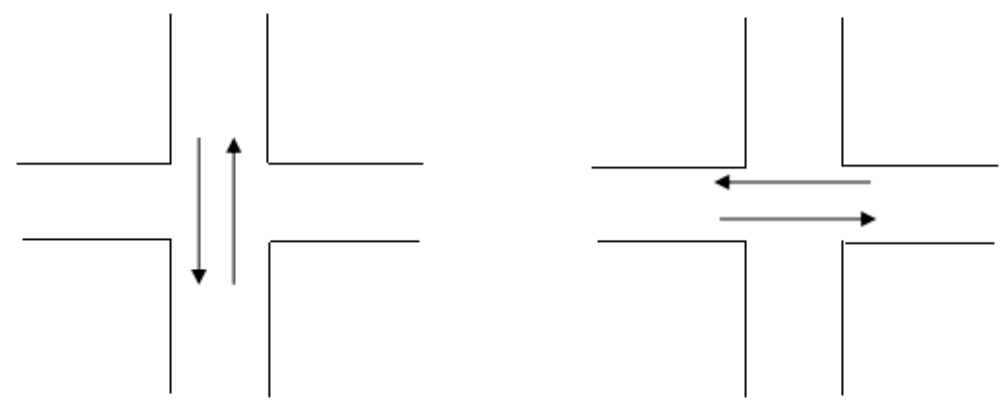

Figure 2 phase traffic diagram for 2

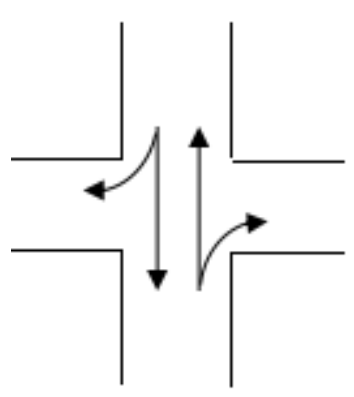

(a)First phase

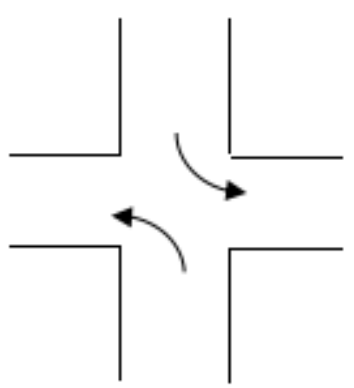

(b) second phase

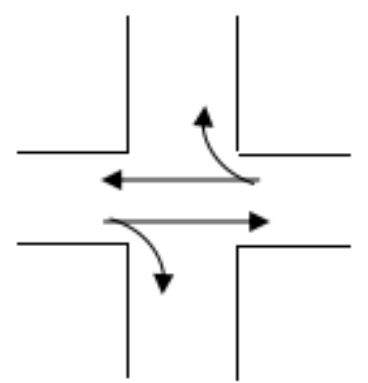

(c) third phase

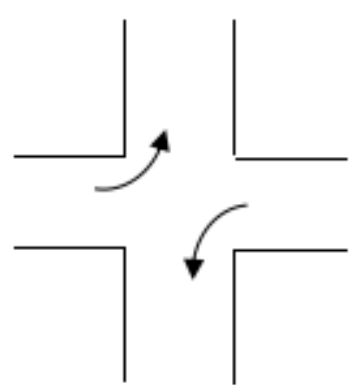

(d) fourth phase

Figure 3 phase traffic diagram for 4

The phase difference is the difference between the starting time of the green light (or the red light) at the same phase of the adjacent intersection. For example, there are two adjacent intersection day east-west street, traffic signal cycle is equal to the same phase (e.g. East-West straight) green (or red) starting time only is the phase difference of the signal intersection things straight. The phase difference is an important control parameter when controlling traffic flow on a main line or traffic flow in a network (i.e., by wire or surface control). By adjusting the phase difference between each intersection, the intersection of trunk line can make a series of lights to form a green belt, so that the team can run in both directions through the intersection of these.

Traffic Driving Model. Vehicle model is the core model of traffic simulation system, and it is the key to evaluate the simulation system. It can be subdivided into the following models: free running model, car following model and lane changing model.

the free running model is simulated where the driver of the vehicle can expect the speed to operate when the vehicle is not in front of the current individual or in the absence of a vehicle 
within its scope of consideration. In addition, under the same conditions, if the vehicle's current speed is less than the expected speed, the driver can take the maximum acceleration and achieve the desired speed in the shortest possible time.

what the car model describes is that the driver wants to approach the vehicle in front as much as possible and has to avoid the mental process of collision with the vehicle in front.

change model is by its own driver driving characteristics, according to the vehicle speed, the gap around the information surrounding the stimulus, adjust and complete their own driving integrated process targets, including the information judgment and execution of two operation process.

\section{Conclusions}

Practice has proved that the intelligent transportation system is an ideal solution to the traffic problems brought about by the current economic development. Research on traffic scheduling modeling for simulation of local intelligent transportation plays a very important role, and can improve the road usage efficiency, reduce vehicle energy consumption, improve traffic congestion, improve transport efficiency and reduce the short current road traffic capacity.

\section{Acknowledgement}

This work was supported by the Liaoning Provincial Social Planning Fund (L15BGL017) and science and technology program of Liaoning(20170540767).

\section{References}

[1] Wang G, Jiang Z, Qian J, et al. A web-based simulation system for traffic evacuation[C]// International Conference on Service Systems and Service Management. 2016:1-6.

[2] Hong Y. The Application Mode of the Technology of the Internet of Things to Urban Intelligent Transportation[J]. Journal of Convergence Information Technology, 2013, 8(7):715-722.

[3] Yin W, Murray P. An agent-based modeling system for travel demand simulation for hurricane evacuation[J]. Transportation Research Part C Emerging Technologies, 2014, 42(42):44-59.

[4] Bakillah M, Zipf A, Liang S H L. Publish/Subscribe System Based on Event Calculus to Support Real-Time Multi-Agent Evacuation Simulation[M]// Geographic Information Science at the Heart of Europe. Springer International Publishing, 2013:337-352.

[5] Zhou H, Liu B, Wang D. Design and Research of Urban Intelligent Transportation System Based on the Internet of Things[M]// Internet of Things. Springer Berlin Heidelberg, 2012:1-3.

[6] Zhang W Y, Wang X F, Feng X. Research on Model-Designing \& Architecture of IOT-Based Intelligent Transportation System[J]. Applied Mechanics \& Materials, 2013, 392:991-996.

[7] Yuan S, Liu Y, Wang G, et al. A Cross-Simulation Method for Large-Scale Traffic Evacuation with Big Data[M]// Web-Age Information Management. Springer International Publishing, 2014:14-21.

[8] Dewen S, Xinhong C, Jing C, et al. Granular Computing in Intelligent Transportation: An Exploratory Study[J]. Cybernetics \& Information Technologies, 2015, 15(6):122-134.

[9] Li N, Huang Z, Xu D, et al. Interface Design of Semantic System for Road Sign Management[M]// Web Information Systems Engineering - WISE 2013 Workshops. 2013:452460. 